\title{
Numerical Studies on the Influence of Step-Like Surface Irregularities on the Development of Tollmien-Schlichting Waves
}

\section{Problem description}

- Flat plate with a step-like irregularity placed at $x_{c}$

- Both forward- (FFS) and backward-facing steps (BFS)

- Mach number $M \boldsymbol{a}_{\infty}=\mathbf{0 . 5}$

- Reference length: Displacement thickness $\delta^{*}$ at $x_{c}$

- Reynolds number based on $\delta^{*}: \operatorname{Re}_{\delta^{*}}=1823$

- Step geometry defined by $z=\frac{H}{2}\left(1+\operatorname{erf}\left(\frac{x-x_{c}}{C}\right)\right)$,

- Step height variation $\boldsymbol{H}= \pm \mathbf{0 . 4}, \mathbf{0 . 8}, 1.2$

- Step shape variation $C=50,10$, rectangular $(C \rightarrow 0)$

- Stability analysis: AHLNS methodology $\widetilde{q}(x, y, z, t)=\widehat{q}(x, z) e^{i \theta}$, where $\theta=\int \alpha d x+\beta y-\omega t$

\section{AHLNS methodology}

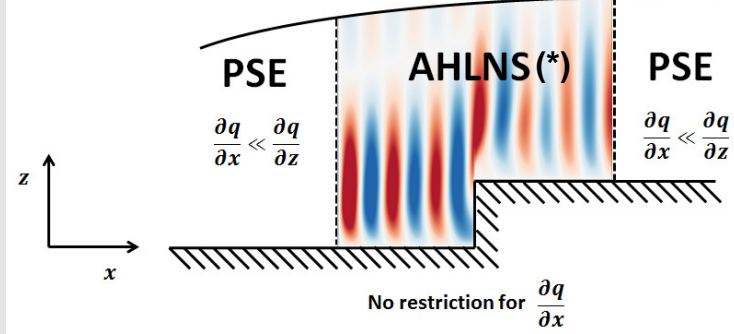

Sketch of the multi-zonal technique for boundary-layer instability analysis in the presence of a FFS. The vertical dashed lines represent the inflow and outflow locations for AHLNS computations. Magnitude $q$ holds for both base flow quantities $\bar{q}$ and amplitude function of the disturbances $\widehat{q}$. Flow direction is from left to right.

$\left.{ }^{*}\right)$ Real part of the streamwise velocity disturbance $\tilde{u}$ computed with AHLNS for the case FFS H08 $r$ and reduced frequency $F=20 \cdot 10^{-6}$.

\section{Base flow computations}

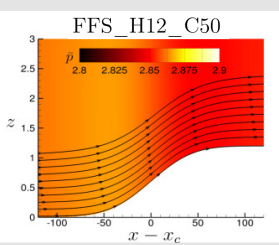

BFS H12 C50

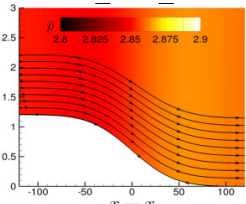

Nondimensional pressure contours $\bar{p}$ and streamlines for FFS and BFS with height $H=1.2$ and shape parameter $C=50,10, r$. Axes are not to scale.

\section{AHLNS validation}

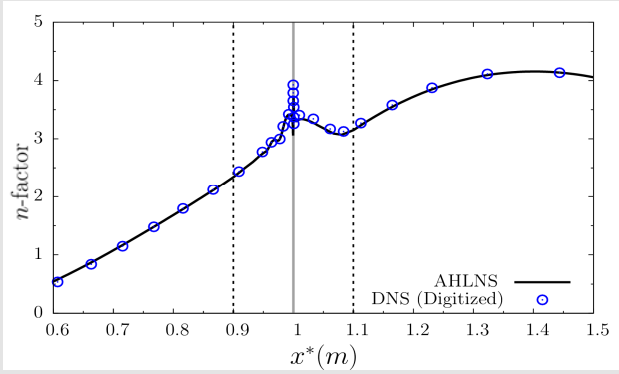

- $M a_{\infty}=0.8$

- $R e_{u}=2.45 \cdot 10^{6} \mathrm{~m}^{-1}$

- $R e_{H}=1320$

DNS: $\sim 300 \mathrm{sec}$. on 6144 proc.

AHLNS: $\sim 300 \mathrm{sec}$. on 4 cores

$n$-factor distribution for an incoming TS wave of reduced frequency $F=21.875 \cdot 10^{-6}$ along a flat plate in the presence of a rectangular FFS. Vertical dashed lines indicate the location of the interfaces between PSE and AHLNS methodologies. The grey line represents the position of the step. DNS points are digitized from the work of Edelmann [1].

[1] C. A. Edelmann, Influence of FFS on laminar-turbulent transition, PhD thesis, Univ. Stuttgart (2014).

\section{Instability analysis: Effect of step height and shape on $\mathrm{N}$-factor envelope curves}

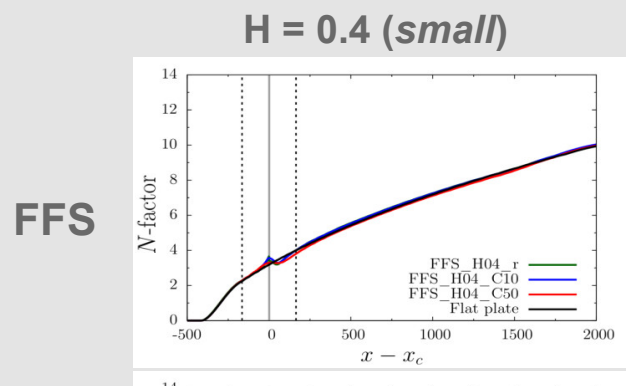

$\mathrm{H}=0.8$ (medium)

$\mathrm{H}=1.2$ (large)

BFS
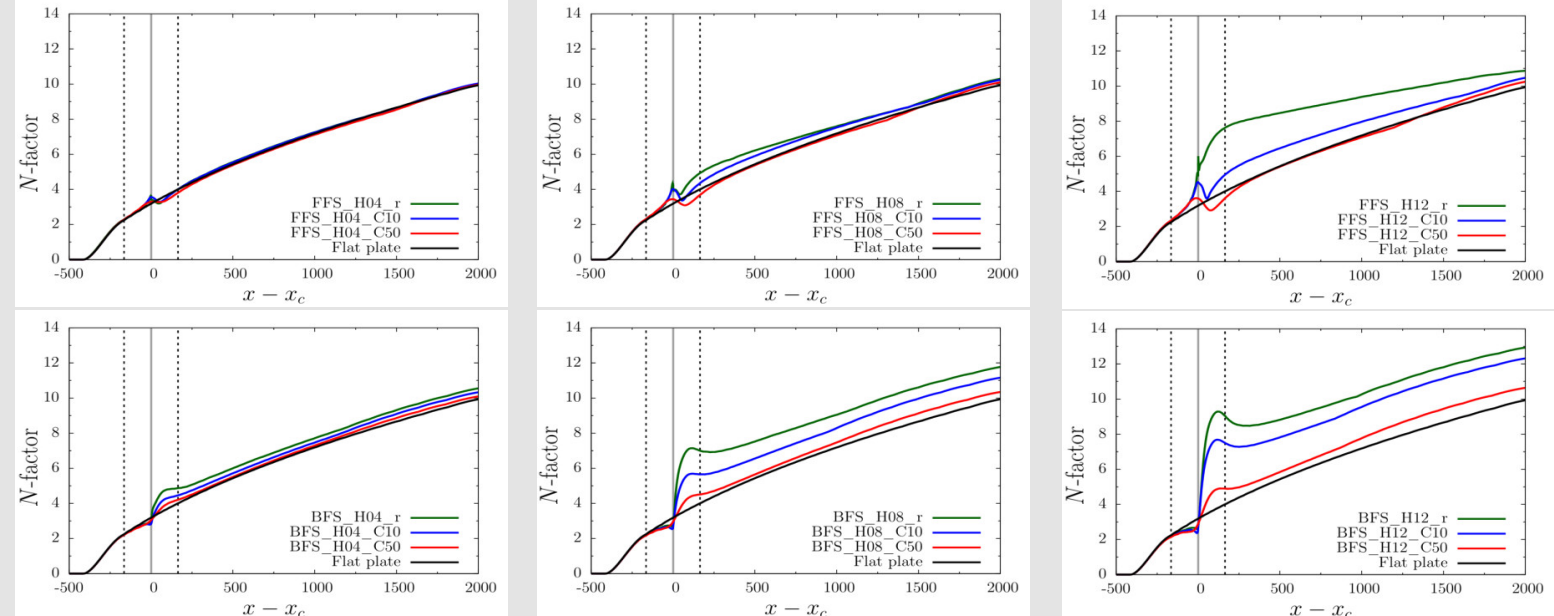

$N$-factor envelope curves, based on streamwise velocity disturbance $\widetilde{u}$, for FFS and BFS with heights $H=0.4,0.8,1.2$ and shape parameter $C=50,10$, Vertical dashed lines indicate the location of the interface between PSE and AHLNS methodologies. The grey line represents the position of the step.

\section{Conclusions}

- Smoothing the shape of medium and large rectangular steps leads to a significant reduction of the $\mathrm{N}$-factor envelope.

- The AHLNS methodology is very well suited for parametric studies applied to surface irregularities.

Juan Alberto Franco Sumariva ${ }^{1,2, *}$ and Stefan Hein 